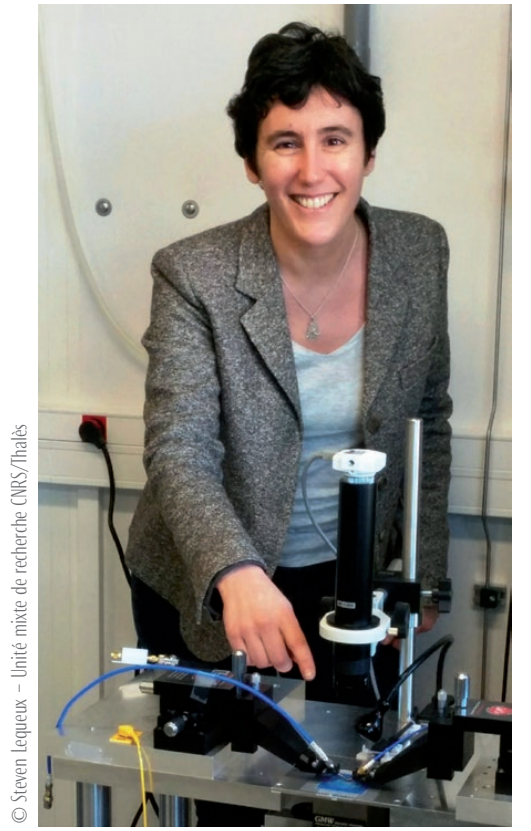

\title{
Julie Grollier, cerveau (spin)tronique
}

Spécialiste de l'électronique de spin, cette touche-à-tout insatiable se passionne pour le fonctionnement du cerveau et vient de prendre la direction d'un groupement de recherche $(\mathrm{GdR})$ du CNRS sur les puces bio-inspirées.

"Ce qui me plait, c'est me servir de la physique que j'aime pour fabriquer des choses. " Ainsi se résume Julie Grollier, chargée de recherches au CNRS, dont la voix à la fois posée et enthousiaste témoigne de la détermination. Celle d'une ancienne élève de l'École supérieure d'électricité qui a découvert la physique par hasard lors d'un stage de recherche au Laboratoire de cristallographie et science des matériaux (CRISMAT), à Caen. " Ça a été la révélation, confie-t-elle. La découverte que la recherche est un espace de liberté et de stimulation intellectuelle sans pareil. "

D'où son intégration à l'École normale supérieure de Cachan en troisième année, suivie d'un master en sciences des matériaux, puis d'un doctorat sous la direction d'Albert Fert. Spécialité ? La spintronique, ou comment utiliser, plutôt que leur charge, la minuscule boussole (le spin) portée par les électrons pour stocker, transmettre ou traiter de l'information.

Précisément, Julie Grollier est alors l'une des premières à mettre en évidence l'effet dit de transfert de spin [1], ouvrant la possibilité de manipuler le spin électronique à l'aide d'un simple courant électrique. De quoi envisager à terme des mémoires non volatiles et très rapides à écrire. "Ce travail m'a beaucoup plu, car il m'a permis de pratiquer aussi bien la salle blanche que la théorie, en passant par des mesures fines de transport électrique", ajoute la physicienne qui revendique un profil éclectique.

Ce travail de thèse a ouvert de nouveaux horizons à cette insatiable curieuse, recrutée en 2005 par le CNRS à l'unité mixte de recherche CNRS/Thalès. D'une part, le développement du transfert de spin dans le domaine hyperfréquence, avec en ligne de mire de possibles applications en télécommunications. D'autre part, sa nouvelle marotte : les puces électroniques dites bio-inspirées, capables de reproduire le fonctionnement du cerveau, avec à la clé : vitesse intrinsèque, tolérance aux défauts et faible consommation énergétique. "Un cerveau consomme 10000 fois moins d'énergie qu'un superordinateur et est imbattable pour des tâches d'interprétation, de synthèse ou de classification ", s'enthousiasme Julie Grollier.
Elle raconte : «En 2009, j’ai découvert les travaux de Hewlett-Packard sur les memristors, de minuscules résistances variables à effet mémoire capables de mimer le fonctionnement des synapses. " Ainsi, grâce à une bourse de l'ERC, elle se lance dans la mise au point de memristors bénéficiant des apports de la spintronique, plus rapides et également plus robustes que les memristors classiques. "Nous avons apporté la preuve du concept sur un memristor unique, et plusieurs brevets ont été déposés."

Empruntant aux neurosciences, à l'informatique, aux mathématiques ou encore à l'électronique ou à la science des matériaux, la suite est affaire d'interdisciplinarité. Ce qui n'est en rien pour effrayer la jeune chercheuse, désormais à la tête du GdR BioComp ( Implémentations matérielles du calcul naturel »), regroupant 52 équipes de 34 laboratoires de cinq instituts du CNRS ! « On est à un moment de convergence très intéressant, et nos discussions visent à déterminer les meilleures voies possibles ", complète la physicienne.

Julie Grollier participe à l'ANR MHANN, dont l'objectif est de fabriquer des réseaux de neurones artificiels avec des memristors ferroélectriques et des composants CMOS. À plus long terme, elle réfléchit également à des solutions à l'échelle nanométrique grâce aux potentialités du transfert de spin. "La spintronique est probablement une approche très prometteuse pour les puces bio-inspirées ", juge-t-elle. La chercheuse est plus déterminée que jamais à utiliser ses talents pour fabriquer des « choses "! !

Mathieu Grousson, journaliste

[1] B. Dieny, Reflets de la physique 16 (2009) 6-11. Web

Site de l'unité mixte de recherche CNRS/Thalès www.trt.thalesgroup.com/ump-cnrs-thales

Site de Julie Grollier

http://julie.grollier.free.fr/publications.htm

Julie Grollier, lauréate ERC 2011

www.cnrs.fr/inp/spip.php?article378 